\title{
Autonomía del paciente y toma de decisiones en salud: conocimiento en internos de medicina - 2010
}

\author{
Patient's autonomy and decision making in health: acquaintance by Peruvian medical
}

interns - 2010

\author{
Cecilia Sogi ${ }^{1}$, Salomón Zavala ${ }^{1}$, Maximiliano Cárdenas ${ }^{1}$, Ana Delgado ${ }^{1}$ \\ 1 Instituto de Ética en Salud, Facultad de Medicina, UNMSM.
}

\begin{abstract}
Resumen
Objetivos: Determinar el conocimiento sobre autonomía del paciente y derecho a participar en las decisiones de salud en internos de medicina, promoción 2010, de cinco ciudades del Perú. Diseño: Estudio tipo encuesta. Institución: Instituto de Ética en Salud, Facultad de Medicina, Universidad Nacional Mayor de San Marcos, Lima, Perú. Participantes: Internos de medicina. Métodos: Se utilizó cinco viñetas de casos de la literatura, con preguntas de selección múltiple como instrumento, el mismo que fue sometido a prueba de validez (juicio de expertos) y confiabilidad (alfa de Cronbach). Participaron en el estudio 272 internos de medicina, promoción 2010, de cinco ciudades del pais (Lima, Ica, Huacho, Trujillo y Huancayo). Principales medidas de resultados: Aciertos en análisis ético. Resultados: Del total de 272 internos, fue varón 61,4\%, edad promedio 26 años (DE 2,8), edad mínima 21 y máxima 47. El número de internos por ciudad fue: Lima $(n=48)$, Huacho $(n=21)$, Ica $(n=24)$, Trujillo $(n=132)$ y Huancayo $(n=47)$. Los aciertos en análisis ético fue alto en los casos $1(74,3 \%), 2$ (68\%) y $5(62,9 \%)$; pero, su aplicación en la decisión fue baja en los casos $1(7,0 \%), 2(3,7 \%)$ y $5(12,5 \%)$. La frecuencia de aciertos fue baja en el caso 3 (análisis ético 23,9\% y decisión clínica 7,7\%). En el caso 4, acertaron en análisis ético $41,9 \%$ y en la decisión, 69,9\%. Conclusiones: Nuestro estudio explora habilidades en situación hipotética. En este contexto, aunque las respuestas sugieren que el análisis ético fue bueno, su aplicación en la decisión fue baja en tres casos, y las respuestas más frecuentes en estos casos sugieren una práctica médica defensiva; asimismo, hay falta de conocimiento sobre consentimiento informado en menores de edad.
\end{abstract}

Palabras clave: Rechazo del tratamiento, análisis ético, decisión clínica, internos de medicina.

\begin{abstract}
Objectives: To determine acquaintance on patient's autonomy and decision making in health care in medical interns from five cities of Peru. Design: Opinion poll type study. Setting: Institute of Ethics in Health, Faculty of Medicine, Universidad Nacional Mayor de San Marcos, Lima, Peru. Participants: Medical interns. Methods: Five cases from the literature with multiple choice questions were used as the instrument; its validity and reliability was established by both a group of experts and Cronbach alpha. Participants in the study were medical interns from five Peruvian cities: Lima $(n=48)$, Huacho $(n=21)$, Ica $(n=24)$, Trujillo $(n=132)$, and Huancayo $(n=47)$. Main outcome measures: Correct ethical answers. Results: From the interns participating 61,4\% were male, mean age 26 (SD 2,8) min 21 and max 47. Correct answers were high in cases $1(74,3 \%), 2(68 \%)$ and $5(62,9 \%)$, but its application in the practice were low in cases 1 $(7,0 \%), 2(3,7 \%)$ and $5(12,5 \%)$. Correct answers were low in case 3 (ethical analysis $23,9 \%$ and decision $7,7 \%$ respectively). In case 4 correct answers were $41,9 \%$ and $69,9 \%$ respectively. Conclusions: In the context of hypothetical clinical situation, participants' answers suggest good ethical analysis but low application in health decision in three cases; the most frequent answers in these cases suggest defensive medical practice. In addition, knowledge about informed consent in minors was low.
\end{abstract}

Key words: Treatment refusal, ethical analysis, clinical decision, medical interns.

An Fac med. 2012;73(1):19-26

La era actual y los vertiginosos cambios sociales que la caracterizan vienen planteando nuevos desafíos a la práctica médica, entre ellas la introducción del principio de autonomía del paciente y su participación en las decisiones relativas a su salud.

El principio de autonomía del paciente ha sido incorporado a la práctica médica a través del consentimiento informado, una contribución del Derecho a la Medicina. Dice Fernández: "Esta inédita situación derivaría en un difícil cambio de actitud del médico frente a su paciente. El facultativo empezaría, lentamente, en un proceso aún inacabado, a tomar conciencia que, a diferencia de lo que hasta entonces había sucedido, se encontraba impedido de invadir y disponer, a su solo arbitrio, el cuerpo ajeno, el de un ser humano que, por ser libre, se le debe suministrar una previa información sobre cualquier acto o procedimiento médico que comprometa su unidad psicosomática” ${ }^{(1)}$.

En la actitud del médico en el encuentro con el paciente, consideramos pertinente mencionar los modelos de toma de decisión en el contexto de salud. Elwyn y col, en 1999, hacen una revisión de la literatura y señalan que el tipo de consulta más frecuente es el modelo paternalista, basado en la concepción de Parson de rol de enfermo. En el otro extremo del espectro ubican la decisión informada, donde la toma de decisión se adjudica enteramente al paciente, con el riesgo de aumentar su ansiedad o hasta hacerlo sentirse abandonado. Y, entre ambos ubica el modelo de decisión participativa (de Charles y col, 1997), cuyas características son: 
a) involucra dos participantes -el médico y el paciente-y a menudo a muchos otros (red familiar y colegas); b) ambas partes (médicos y pacientes) toman acciones para participar en la toma de decisión; c) la información compartida es un prerrequisito; d) se toma una decisión de tratamiento (incluyendo el no tratamiento) y ambas partes aceptan la decisión ${ }^{(2)}$.

La participación del paciente en la decisión no siempre es el mejor modelo en una consulta médica, dependiendo de sus características o el contexto de la consulta. Así, Savage y Armstrong hallaron que el estilo directivo tuvo mayor efecto en términos de satisfacción del paciente. Los autores plantean una posible relación entre estilos de consulta con dos grandes grupos de enfermedades. Aquellas de naturaleza física simple, que responden a la aproximación biomédica tradicional de diagnóstico y tratamiento, parecen beneficiarse del estilo mencionado. Sin embargo, en otros problemas, particularmente enfermedades crónicas y psicológicas, con mayor componente psicosocial, que el modelo biomédico maneja pobremente, ese beneficio desaparece ${ }^{(3)}$. Adicionalmente, otras características del paciente que es probable que ejerzan un efecto modificador son el nivel de educación, severidad de la enfermedad y mayor edad, aunque ninguna variable es predictiva ${ }^{(2)}$.

Las particularidades de la naturaleza humana se reflejan en el hallazgo de Charles y col (citados por Elwyn y col), que la relación entre "preferencias del paciente a participar" y la participación efectiva no son consistentes. Por ejemplo, la mayoría de sujetos sanos opinan a favor de elegir su propio tratamiento; sin embargo, de aquellos que tienen cáncer, solo una minoría comparte la misma opinión ${ }^{(2)}$.

De otro lado, el modelo de decisión participativa, dicen Brock y Wartman, simplifica demasiado las complejidades de roles y contribuciones de médicos y pacientes cuando se toma decisiones de tratamiento reales, aunque destaca la participación activa de este último en el proceso. Los autores proponen una taxonomía de las diferentes fuentes y formas de decisiones irracionales en la negativa del paciente al tratamiento:

- Sesgo orientado al presente y futuro inmediato. Las personas generalmente dan mayor peso en asegurarse beneficios y evitar riesgos en el presente y futuro inmediato que en un futuro remoto.

- "Esto no me pasará a mí". Pacientes que niegan riesgos o tienen creencias mágicas sobre su vulnerabilidad al riesgo o tienen una forma diferente de ver el problema médico.

- Miedo al dolor o a la experiencia médica. Los pacientes son diferentes, por ejemplo, en la medida en que están preparados para tolerar tratamientos o condiciones penosas en bien de otros fines, que hay que diferenciar del peso indebido que atribuyen algunos a aspectos del tratamiento por miedos irracionales.

- Lo que el paciente quiere no tiene sentido. Frecuentemente esto ocurre con los Testigos de Jehová. En otros casos, aquello que decide el paciente no tiene sentido, p. e., puede decir que comprendió la información pero que simplemente no le importa morir o quedar incapacitado por no recibir el tratamiento. En estos casos, lo difícil es determinar la autenticidad de la decisión y si se trata de una distorsión de valores causada por una condición tratable, tal como una depresión.

- Efectos de cómo se presenta la información. Lo mejor que puede hacer el médico es presentar la opción de modo alternativo, con la esperanza de disminuir el efecto del fraseo.

- Responsabilidad social versus individual: a) uso irracional de recursos. Un paciente asegurado tiene poco o nada que evaluar respecto a los costos reales contra los beneficios esperados. Cuando al paciente no les cuesta del bolsillo, es racional para ellos elegir toda la atención médica sin importarles el costo; b) salud pública versus salud individual, p. e., la vacunación que beneficia la salud pública más no necesariamente la individual (4).

Con relación a la toma de decisiones en salud, tal como sostiene Pellegrino: "La condición moralmente óptima es aquella en la cual la decisión proviene del médico y el paciente. Por su parte, el médico debe tomar la decisión por y con el paciente, por no significa en lugar del paciente, sino por sus intereses. Esta formulación preserva el derecho legal a la integridad, el derecho moral a la autonomía y el derecho moral más profundo a la integridad de las personas".

El autor citado señala deficiencias de la autonomía como guía moral: "La autonomía ha llegado a tener una acentuada cualidad legalista, centrada con demasiada frecuencia en las leyes generales relativas a la invasión de la intimidad. Este enfoque promueve la frecuente noción de la relación del médico y el paciente como un contrato, más que como una relación fiduciaria o de acuerdo... Por otro lado, la gran importancia dada a la autodeterminación minimiza las obligaciones del médico respecto a la beneficencia y a la renuncia al propio interés" ${ }^{(5)}$.

Por su lado, Quill y Brody sostienen que: "El tema filosófico central de la autonomía es el respeto hacia el paciente como persona. No es respeto privar a las personas de las recomendaciones médicas para mantener la neutralidad, ni tampoco tratarlos de acuerdo a protocolos rígidos, sea para tratamiento agresivo como para el paliativo. Respetar a una persona significa darse tiempo para escuchar la historia personal y asegurarse de que las decisiones médicas se integran a la biografía del paciente en el momento actual" ${ }^{(6)}$.

La toma de decisiones médicas no siempre es una tarea sencilla y, hoy en día, existen programas que intentan promover la participación del paciente, en alcanzar una decisión informada. El modelo mencionado de decisión parti- 
cipativa ha sido motivo de estudios que evalúan su eficacia. Así, $\mathrm{O}^{\prime}$ Connor y col hallaron que el modelo es mejor que la atención médica estándar en mejorar conocimiento, confort y participación en la decisión sin aumentar la ansiedad. Sin embargo, tiene poco efecto sobre la satisfacción y efecto variable en la decisiones de los pacientes; asimismo, cuando la ayuda del paciente es más detallada, aunque mejora su confort en la toma de decisión, su conocimiento solo mejora marginalmente ${ }^{(7)}$.

Respecto a la capacidad de decisión (competencia), en una situación clínica la capacidad de un paciente para consentir o rechazar un tratamiento requiere de habilidad para comprender la información relevante, evaluar la situación médica y sus posibles consecuencias, para comunicar una elección y participar en una deliberación racional. En otras palabras, la capacidad de decisión se refiere a acciones específicas de comprender, evaluar y hacer una elección entre opciones realistas. Determinar esta capacidad es tarea del clínico ${ }^{(8)}$.

La competencia que es el término legal de capacidad de decisión utiliza frecuentemente como criterios: habilidad para comunicar la elección, comprensión de la información sobre tratamiento, apreciación de la situación y sus consecuencias. Sin embargo, utiliza menos frecuentemente la deliberación -uso del proceso lógico para evaluar riesgos y beneficios de las opciones terapéuticas-, habilidad que puede estar alterada en trastornos mentales y emocionales ${ }^{(9)}$.

De otro lado, en el encuentro médico paciente, como en toda relación humana, se producen inevitablemente respuestas emocionales. Zinn señala que el estado psicológico y emocional del paciente estimula la respuesta correspondiente en el médico; por ello, tales respuestas deben ser analizadas averiguando su significado. Agrega que, respecto a la respuesta emocional como información acerca del médico, constituye un problema si ella interfie- re con el diagnóstico y tratamiento del paciente ${ }^{(10)}$.

A lo mencionado se suma la necesidad de considerar otros criterios como los valores y metas del propio paciente. $\mathrm{Al}$ respecto, Huijer y van Leeuwen llevaron a cabo un estudio en pacientes con cáncer que rehusaron tratamiento y sus oncólogos. Hallaron que estos últimos concluyeron que los pacientes tomaban una decisión irracional si, después de balancear los riesgos y beneficios, no optaban por el curso de acción con mayores beneficios. La racionalidad de la decisión fue dirigida por los valores médicos tales como: posibilidad de recuperación, prolongar la vida o aliviar síntomas. La entrevista con pacientes mostró que aquello que parece tonto en el contexto médico, en realidad era resultado de un proceso de balance en el contexto personal del paciente. La negativa pudo ser mejor entendida en el marco de una visión más amplia de su mundo. En este contexto: valores personales, emociones, creencias y actitudes hacia la vida, sufrimiento y muerte, parecen proporcionar buenas razones para que el paciente rehúse tratamiento ${ }^{(11)}$.

En el país, entre las pocas publicaciones sobre el tema de la autonomía del paciente, existe uno del Comité de Bioética del Consejo Nacional de Salud (2009). En esta se señala que: "Muchos pacientes, por diversas razones, pueden plantear demandas irracionales antes o después de los procedimientos, como es el caso de pacientes con costumbres ancestrales de escasa comprensión por la medicina occidental y la incompatibilidad idiomática que no puede expresar con precisión los objetivos propuestos. Ello ocurre sobre todo en casos de emergencias o urgencias calificadas, pacientes terminales, pacientes con alteraciones neurológicas o psiquiátricas, y en quienes resulta difícil establecer la capacidad de comprensión debido a severo estrés o shock ${ }^{(12)}$.

El objetivo del presente estudio fue evaluar el análisis y la decisión ética en casos hipotéticos, en internos de medicina.

\section{MÉTODOS}

Los participantes en el estudio fueron 272 internos de medicina, promoción 2010, de cinco ciudades del país: Lima $(\mathrm{n}=48)$; Huacho $(\mathrm{n}=21)$; Ica $(\mathrm{n}=$ 24); Huancayo $(n=47)$ y Trujillo ( $=132$ ).

Para determinar el instrumento, se revisó la literatura sobre el tema de estudio y se eligió cinco viñetas de casos ${ }^{(8,13-15)}$. Luego, se elaboró once preguntas de selección múltiple. Al final se pidió comentarios sobre el cuestionario y anotar el sexo, edad, universidad de formación y año de egreso. En los resultados se presenta las viñetas, con sus respectivas preguntas.

La validez del instrumento se estableció por medio del juicio de expertos. Se elaboró un cuestionario para los jueces indagando si las preguntas, así como las opciones de respuesta, eran o no precisas. Si la respuesta era negativa, se solicitó que lo comentara. Participaron ocho jueces, médicos del Instituto de Ética en Salud de la UNMSM. En la prueba binomial, la concordancia entre jueces osciló entre $\mathrm{p}<.05-\mathrm{p}<$ .001 , excepto en las preguntas 7 y 9 ; sin embargo, por interés de la investigación estas fueron mantenidas. La confiabilidad se estableció con el total de participantes en el estudio y se utilizó la prueba alfa de Cronbach 0,23.

En cada ciudad se estableció contacto con profesores de la universidad local. Se les explicó los objetivos del estudio, solicitando su colaboración. Los datos fueron recolectados en el segundo semestre del año 2010.

Para el análisis estadístico, los cuestionarios fueron revisados para separar aquellos incompletos y luego se elaboró la base de datos en Excel. Se utilizó el paquete estadístico SPSS versión 15 para análisis de frecuencia y chi cuadrado.

Con relación a la ética, se comunicó verbalmente a los internos que la participación era voluntaria, anónima y que los resultados serían utilizados para los propósitos del presente estudio. 


\section{RESULTADOS}

Participaron en el estudio 272 internos de medicina, promoción 2010, de cinco ciudades del país (Lima, Ica, Huacho, Trujillo y Huancayo). El 61,4\% fue varón; la edad promedio fue 26 años (DE 2,8), mínima 21 y máxima 47.

A continuación se muestra los casos presentados a los alumnos, las preguntas y las respuestas éticas.

\section{Caso 1.}

Anciana de 76 años de edad que llega a la sala de emergencia por dolor retroesternal opresivo, desencadenado por el ejercicio y calma con el reposo. Hace dos semanas el dolor aumenta en frecuencia e intensidad. El médico indica hospitalización por riesgo de infarto agudo del miocardio y muerte. Paciente relata que hace seis meses falleció su esposo luego de una infructuosa reanimación cardio-pulmonar. Recuerda que su cónyuge fue intubado a pesar de su negativa y ella no desea pasar por lo mismo y prefiere morir y reunirse con su esposo.

$(\mathrm{n}=272)$

P1. ¿Cuál considera que es el problema ético más importante?

Respuesta: Respeto a la autonomía de la paciente. $74,3 \%$

P2. ¿Cómo enfrentaría usted el caso?

Respuesta: Respetaría la decisión de la paciente. $7,0 \%$

\section{Caso 2.}

Varón de 24 años de edad, es traído a la sala de emergencia por un amigo. Previamente sano, empezó a quejarse de severo dolor de cabeza y rigidez de nuca. El resultado del examen físico y de laboratorio, incluyendo análisis del LCR, sugieren diagnóstico de neumonía y meningitis por neumococo. Cuando se le informa el diagnóstico y le dicen que será hospitalizado para tratamiento antibiótico, el paciente rechaza la indicación médica sin dar razones.

$(\mathrm{n}=272)$
P3. ¿Cuál es la naturaleza de este caso?

Respuesta: Hay un conflicto entre los principios de autonomía y beneficencia. $68,0 \%$

P4. En este caso, el médico explica las consecuencias funestas del no tratamiento así como los riesgos mínimos de este tratamiento, pero él persiste en su negativa. Aparte del rechazo al tratamiento, el paciente no muestra alteración de su estado mental. ¿Cuál sería su juicio ético?

Respuesta: No existe evidencia de incapacidad mental. 26,1\%

P5. Frente a la negativa al tratamiento, ¿qué curso de acción tomaría usted?

Respuesta: Procedo con el tratamiento contra los deseos del paciente. 3,7\%

\section{Caso 3.}

Adolescente varón de 14 años de edad padece de leucemia linfocítica, tiene su segunda recaída y no responde a la quimioterapia. Sufre de anemia y trombocitopenia. Él comprende que la transfusión sanguínea lo aliviaría, disminuyendo la posibilidad de hemorragias que pondrían en peligro su vida. Él reafirma su fe como Testigo de Jehová y rechaza la transfusión, y sus padres coinciden con la decisión.

$(\mathrm{n}=272)$

P6. ¿Cuál es la naturaleza del caso?

Respuesta: Menor de edad rechaza tratamiento indicado por motivos religiosos. $23,9 \%$

P7. ¿Cuál sería el curso de acción que seguiría usted?

Respuesta: Evalúo la comprensión de las consecuencias de la decisión. 7,7 \%

\section{Caso 4}

Adolescente mujer de 15 años consulta para un examen médico anual. Su rendimiento académico es regular, pero es muy popular en su clase y participa en actividades extracurriculares. El examen médico fue normal, pero ella le cuenta al médico que recientemente se ha enamorado de un chico de 16 años. Su padre piensa que aún es muy joven y le prohibe salir con el chico. Ella afirma que aún no es sexualmente activa, pero pide le prescriban píldoras anticonceptivas por si acaso, sin comunicarlo a sus padres, porque se molestarían. Ella parece ser madura y razonable

$(n=272)$

P8. ¿Cuál cree usted que sería el problema ético más importante?

Respuesta: Principio de autonomía en menor de edad. $41,9 \%$

P9. ¿Cómo enfrentaría usted el caso? Respuestas:

Accedería al pedido de la paciente, prescribiendo anticonceptivos. 5,9\%

Educaría a la paciente sobre su sexualidad (riesgo de embrazo e ITS). 69,9\%

\section{Caso 5.}

Varón de 22 años en estado vegetativo persistente por TEC grave, en un servicio público para pacientes crónicos. Una semana después del accidente, los padres aceptaron alimentación por gastrostomía a condición de que sería retirada si el pronóstico de recuperación era muy pobre. Un año después los padres solicitan al médico descontinuar la alimentación artificial, pero para su sorpresa, el médico se rehusa aduciendo que este acto sería equivalente a un crimen.

$(n=272)$

P10. ¿Cuál es el tema ético más importante?

Respuesta: Derecho de los padres a decidir por su hijo. $62,9 \%$

P11. ¿Cómo enfrentaría usted el caso? Respuesta:

Indicaría que se retire el tubo de alimentación por ser fútil. 12,5 \%

El puntaje promedio de aciertos fue 4,02 (DE 1,5), para un puntaje máximo de 11; puntaje mínimo 0 y máximo 8 aciertos. Adicionalmente el puntaje 
total de aciertos se clasificó en tres: 0-3 aciertos, 102 (37,5\%); 4-5 aciertos, 130 $(47,8 \%) ; 6+40(14,7 \%)$.

Para fines de análisis bivariado, la variable sexo fue agrupada en varones y mujeres; y la edad en 25 años y menores, y 26 años y mayores. Significativamente más varones que mujeres acertaron en P10 ( $\mathrm{p}<, 01)$; y, en la misma pregunta 10 , acertaron más los de 26 años y mayores que los de 25 y menores $(\mathrm{p}<, 05)$.

Resumiendo nuestros hallazgos, los aciertos fueron altos en el análisis ético de casos 1 (74,3\%), 2 (68\%) y 5 $(62,9 \%)$. Sin embargo, la aplicación del análisis en la decisión clínica fue baja, en los casos 1 (7,0\%), 2 (3,7\%) y $5(12,5 \%)$. La frecuencia baja de aciertos en el caso 3 (análisis ético 23,9\% y decisión clínica 7,7\%) indicaría falta de conocimiento sobre consentimiento informado en una menor de edad. Respecto al caso 4 , acertaron en el análisis ético $41,9 \%$ y en la decisión un porcentaje mayor $(69,9 \%)$.

Cabe mencionar que, respecto a la decisión clínica, las respuestas más frecuentes fueron: en el caso $1(44,1 \%)$ "que firme documento de exoneración de responsabilidad por su negativa"; caso 2 (51,8\%) "solicito autorización legal"; casos $3(57,4 \%)$ y $5(42,7 \%)$ "solicito la intervención del fiscal", que sugieren una actitud defensiva, aunque en casos hipotéticos. Finalmente, hubo internos que no identificaron temas éticos en las viñetas, aunque poco frecuentes, en los casos 1 (3,3\%), 2 $(6,3 \%), 4(12,5 \%)$ y $5(5,9 \%)$.

\section{DISCUSIÓN}

\section{Caso 1.}

Consideraciones éticas. El autor del caso señala que la paciente tenía capacidad de decisión (competencia); su negativa reunió los requisitos de un rechazo informado; y las razones de su renuencia eran consistentes y compatibles con su historia biográfica ${ }^{(13)}$. En otras palabras, el médico respetó la integridad de la paciente, reconociendo su autonomía y comprendiendo su negativa a ir al hospital y aceptar procedimientos agresivos. Pellegrino, en su artículo 'La relación entre la autonomía y la integridad en la ética médica', establece la diferencia entre autonomía, como capacidad inherente al hecho de ser persona racional, e integridad, como atributo de la condición humana. "Respetar la integridad implica tomar en cuenta la decisión de una persona entera o completa". De otro lado, "el médico que hace eso tiene integridad, entereza moral y respeta los matices y las sutilezas del derecho moral a la autonomía” (5).

Por su lado, Beauchamp y Childress (pp. 181), señalan que: "Causar la muerte de una persona es moralmente reprobable, porque una intervención no autorizada obstruye o menoscaba los intereses de ella; privarla de oportunidades y beneficios es un acto no justificado. Sin embargo, si una persona autoriza libremente su muerte y hace un juicio autónomo, el cese del dolor y sufrimiento a través de esa acción constituye un beneficio personal más que un menoscabo a sus intereses. Luego, ayudarlo a morir atendiendo su petición no es pernicioso ni erróneo. Por el contrario, no ayudarla con su cometido frustrará sus planes, dañándola, causándole indignidad y desesperanza. En esta perspectiva, causar la muerte no siempre es un acto malo" (16).

Consideraciones legales. En el país, Fernández sostiene que el proceso de actualización y perfeccionamiento del Código Civil después de veinticinco años de vigencia, está a punto de restablecer aquel primer párrafo del enmendado artículo 11: "Toda persona puede negarse a ser sometida a un examen o tratamiento médico, salvo disposición legal que establezca su obligatoriedad dentro de los límites del respeto a la persona humana. No cabe violentar la libertad personal con el propósito de someter a la persona a un examen o tratamiento médico por ella rechazado, aún en el indeseado supuesto de que dicha omisión acarrearía un grave daño a la salud o acabaría con la salud del paciente" ${ }^{(1)}$.

Adicionalmente, la modificatoria a la Ley general de salud, de 2009, en su artículo 15.2 Acceso a la información, inciso g), también reafirma lo mencionado ${ }^{(17)}$.

\section{Caso 2.}

Consideraciones éticas. El autor del caso señala que la capacidad de decisión tiene niveles dependiendo de la severidad de la enfermedad y la urgencia del tratamiento. Por ejemplo, un paciente necesita alcanzar un estándar mínimo de capacidad para consentir un procedimiento con beneficio alto y riesgo bajo, tal como antibióticos para meningitis bacteriana ${ }^{(8)}$.

Beauchamp y Childress (pp. 131) sostienen que en casos de falsas creencias por ignorancia, que obstaculiza una decisión informada, puede ser permisible y aún probablemente compulsorio, promover la autonomía tratando de imponer una información no deseada. Pone como ejemplo el caso de una mujer con carcinoma de cérvix, estadio $1^{\underline{a}}$, que rechaza la cirugía; y los médicos consideran pasar por alto tal rechazo, porque la sólida evidencia médica demuestra que la paciente no tenía razones para creer que no tenía cáncer. Adicionalmente, los mismos autores (pp. 216) señalan que el paternalismo fuerte se justifica si se satisface las siguientes condiciones ${ }^{(16)}$ :

1. Un paciente está en riesgo de un daño significativo y prevenible

2. La acción paternalista probablemente evitará el daño

3. El beneficio esperado para el paciente a través del paternalismo sobrepasa los riesgos

4. No existe alternativa razonable a la limitación de la autonomía

5. Se adopta la alternativa que menos limite la autonomía, que asegurará los beneficios y limitará los riesgos 
6. La acción paternalista no dañe los intereses substanciales de la autonomía (p. e., creencias religiosas).

Por su lado, Diego Gracia dice, el principio de beneficencia se une con el de no maleficencia en un solo principio, "que mande no hacer mal a nadie y promueva el bien. Así entendido el principio de beneficencia, no hay duda de que ha sido y sigue siendo el santo y seña de la ética médica. Las dos partes de este principio están perfectamente documentadas desde el tiempo de los hipocráticos. La parte negativa se halla formulada en la máxima primum non nocere; la segunda, en la expresión, presente por dos veces en el Juramento hipocrático: "en beneficio del enfermo"... Ambas fórmulas están de algún modo sintetizadas en el criterio del libro de las Epidemias: favorecer o al menos no perjudicar" ${ }^{(18)}$.

Consideraciones legales. En el Perú, la Ley general de salud (1997), en el Título 1 de los derechos, deberes y responsabilidades concernientes a la salud individual, artículo 3 dice: Toda persona tiene derecho a recibir, en cualquier establecimiento de salud, atención médico-quirúrgica de emergencia cuando la necesite y mientras subsista el estado de grave riesgo para su vida o su salud ${ }^{(17)}$. Y, en el artículo 4, respecto al consentimiento previo al tratamiento médico-quirúrgico señala que se exceptúa de este requisito las intervenciones de emergencia. En el caso 2, por su severidad y urgencia de tratamiento, estaría exceptuado del consentimiento informado.

\section{Caso 3.}

Consideraciones éticas. Los autores del caso señalan que, en casos de Testigos de Jehová, se debe respetar el rechazo a la transfusión. Y, como regla general, los deseos de un menor maduro deben ser considerados seriamente en las discusiones relativas a su cuidado. Asimismo, buscar signos de que el menor comprende la situación y valora sus consecuencias ${ }^{(8)}$.
El Comité de bioética de la Academia Americana de Pediatría (AAP) señala que la legislación y la política de la AAP reconocen la doctrina del menor maduro. Este concepto admite que muchos menores, usualmente en los inicios de su adolescencia, pueden contribuir con las decisiones médicas, incluyendo aquellas de soporte vital. Así, en casos particulares, se puede evitar disputas cuando el menor tiene la capacidad de tomar una decisión independiente, a la luz de los valores religiosos y el tratamiento médico recomendado ${ }^{(19)}$.

Consideraciones legales. En el país, Fernández señala que las legislaciones vigentes fijan en catorce o dieciséis años la edad para prestar personalmente su consentimiento informado. Dice: "Compartimos la opinión de un sector de la doctrina que estima que la decisión atinente a su vida o a su salud corresponde al menor mayor de catorce años de edad, siempre que se compruebe, fehacientemente, que es intelectual y emocionalmente maduro para adoptar decisiones por sí mismo... Estimamos que por respeto a la dignidad inherente a la persona del menor, no se puede dejar de lado el derecho de aquellos mayores de catorce años, que están en condiciones de decidir sobre sus derechos fundamentales. Debemos, siempre y en lo posible, respetar dicha dignidad que, como se ha señalado, se sustenta en la libertad y la identidad que son inherentes a la persona y que hace a cada uno de ellas un ser único, singular, irrepetible. Si esto es así, no se debe tratar a todos los seres humanos de la misma manera, sino, más bien, atendiendo y respetando su singularidad. Por ello, no se puede generalizar e impedir que algunos menores de edad mayores de catorce años, que demuestren madurez intelectual y emocional, actúen como lo que son, es decir, como seres libres capaces de decidir por sí mismos" ${ }^{(1)}$.

\section{Caso 4.}

Consideraciones éticas. Los autores del caso, Friedman y Lantos, dicen que: En los EE UU, los menores tienen dere- cho legal para tomar decisiones de salud bajo leyes de consentimiento especial, independientemente de la opinión personal del profesional sobre la autoridad de los padres y la posición moral de aquellos para tomar sus propias decisiones en salud. Estas leyes varían en amplitud, pero todas dan autonomía legal a los adolescentes para buscar y aceptar, por ellos mismos, diagnóstico y tratamiento de abuso de alcohol y drogas; y, consejería y prescripción de anticonceptivos. Algunos Estados permiten a los menores consentir el aborto sin conocimiento y aceptación de sus padres.

Los autores plantean la pregunta: ¿Médico y menor tienen derecho a la confidencialidad, excluyendo a los padres? Y, responden que la respuesta no es unánime. Si los padres rechazan una relación confidencial, los adolescentes necesitan saberlo y el diálogo se debe limitar a aquellos temas que ellos quieren compartir con sus padres. Sin embargo, aún cuando se establezca una relación confidencial, el médico debe explicar al adolescente que existen limitaciones. Por ejemplo, el médico puede romper el secreto si sospecha que el adolescente es suicida o víctima de abuso familiar. Luego, es necesario decidir si el adolescente es competente para tomar decisiones en temas sexuales y reproductivos, y si ella es suficiente para concederle autonomía en la atención de su salud. Uno puede prescribir anticonceptivos por razones prácticas, aún cuando el adolescente no es competente, sin embargo, no existe consenso a este respecto ${ }^{(14)}$.

Consideraciones legales. En el Perú, la Ley general de salud (1997), en su artículo 6, señala que: Toda persona tiene derecho a elegir libremente el método anticonceptivo de su preferencia ${ }^{(17)}$. No menciona la edad a partir de la cual puede ejercer ese derecho. Sin embargo, si las legislaciones vigentes fijan en catorce o dieciséis años la edad para prestar su consentimiento informado ${ }^{(1)}$, si el menor es maduro, ipodría ejercer su derecho a solicitar un método anticonceptivo? 


\section{Caso 5.}

Consideraciones éticas. Los autores del caso, Bascom y Tolle, señalan que en los Estados Unidos existe consenso en relación a los estándares profesionales para una práctica ética en muchos aspectos de la atención al final de la vida, producto de mucha discusión, debate y casos judiciales. Aspectos en los que existe claridad ética y legal, aún cuando surgen casos que pueden desafiarlos, incluyen la capacidad de pacientes o sus familiares de rechazar tratamientos de soporte vital, incluyendo la nutrición e hidratación artificial, la aceptabilidad ética tanto de iniciar como suspender tratamientos de soporte vital, y el derecho de los pacientes de recibir dosis altas de analgésicos, aún cuando ello conlleva el riesgo de acortar la vida. De otro lado, persiste el debate acerca de la ética del suicidio asistido, que continúa siendo ilegal en muchos Estados... En cada uno de estos aspectos de la práctica médica, el proceso de toma de decisión continúa siendo emocionalmente cargado e intrigante para médicos, paciente y familia.

Los mismos autores, respecto al caso, relatan que: El médico rechaza suspender la alimentación artificial aduciendo que tal acto viola sus propias creencias morales. Los padres solicitan ayuda para localizar otro médico que acepte su petición; sin embargo, esta solicitud también fue negada. Finalmente, los padres contactaron con el hospital, donde le colocaron la sonda para la alimentación. El servicio de ética del hospital ayudó a ubicar un médico, quien respetó la decisión de los padres. La alimentación artificial fue retirada y el paciente falleció a las dos semanas ${ }^{(15)}$.

Beauchamp y Childress (pp. 132), respecto a la decisión de sustitución, recomiendan la necesidad de considerar cada caso por separado. Sin embargo, puede ser adecuado contar con procedimientos establecidos. Por ejemplo, las instituciones podrían establecer reglas que desaprueben la decisión de sustitución, excepto cuando ella ha sido aprobada por comités de revisión institucional o de ética asistencial ${ }^{(16)}$.

En la decisión de sustitución en pacientes incompetentes, Emmanuel y Emmanuel (1992), sostienen que: Históricamente, la justificación para este tipo de decisión es que: 1) el derecho del paciente incompetente a rehusar tratamiento médico puede ser ejecutado a través de una decisión de sustitución; 2) conociendo bien los deseos del paciente, el sustituto tomaría la decisión que elegiría el paciente de ser competente. En la doctrina jurídica esto se llama juicio sustituto. No obstante, existen objeciones teóricas y empíricas que cuestionan esta decisión, en el sentido de si ella alcanza el objetivo de promover las preferencias del paciente respecto a su tratamiento ${ }^{(20)}$.

La President's Commission y otros sostienen que, aunque los sustitutos no puedan predecir con precisión las preferencias del paciente, la política social debe respetar la buena fe de las decisiones familiares basadas en los mejores intereses del paciente. De otro lado, Buchanan advierte que, si bien el consenso social y la Suprema Corte reconocen el derecho del paciente a rehusar tratamiento, esto no tiene peso para justificar el derecho de la familia a tomar decisiones por el paciente, basadas en sus mejores intereses. Este derecho necesita de una justificación independiente...; nuestra sociedad ha vacilado en investir a la familia de autoridad plena sobre sus miembros, aún en los casos de menores y adultos incompetentes...; cuando la familia no es guiada por preferencias explícitas del paciente, sus decisiones deben ser limitadas y supervisadas ${ }^{(20)}$.

Consideraciones legales. Respecto a la decisión de sustitución en pacientes incapaces, Fernández revisa las declaraciones internacionales y la legislación nacional. Menciona como preocupación que el derecho a rehusar tratamiento se extienda a su representante o a una persona legalmente designada para el efecto, "si de lo que se trata es de mejorar la salud o salvar la vida del incapaz, solo la persona, por sí misma, estaría en condiciones de adoptar tan grave decisión, motivadas por razones subjetivas ajenas al conocimiento de terceros". Cita el ejemplo del caso Quinlan de la jurisprudencia norteamericana, en el que el Tribunal Supremo del Estado de Nueva Jersey determina como la única manera práctica de prevenir que se le destruya su derecho a la intimidad de la paciente a terminar con su vida, debido a su existencia vegetativa, no cognoscitiva, es admitiendo que su tutor o familia ejerzan su mejor criterio, es decir, tal como la propia paciente lo hubiera ejercido en las mismas circunstancias ${ }^{(1)}$.

Respecto a nuestros hallazgos del presente estudio del conocimiento sobre autonomía y toma de decisión en salud, de los internos de medicina, promoción 2010, de cinco ciudades del país, no existen publicaciones previas que permitan una discusión. El único estudio sería el nuestro, en médicos postulantes a las plazas de residencia de la Universidad Nacional Mayor de San Marcos, 2010. En esta población se examinó el conocimiento sobre autonomía del paciente y derecho a rehusar un tratamiento recomendado, utilizando los mismos casos 1,2 y $3^{(21)}$.

En la comparación de ambas poblaciones (casos 1-3), los internos de medicina y los médicos, pese a diferencias en edad promedio -26 y 31 años, respectivamente-, y por consiguiente, diferencias en tiempo de experiencia clínica, sus respuestas fueron similares. Es decir, en los casos 1 y 2 se observó discordancia entre análisis y decisión ética, así como frecuencia alta de respuestas que sugieren una actitud defensiva, al menos en situación clínica hipotética, en ambas poblaciones. En el caso 3, la frecuencia baja de aciertos en el análisis y decisión ética sugieren desconocimiento del tema consentimiento informado en menores de edad, en ambas poblaciones.

La principal limitación del estudio sería la confiabilidad del instrumento, que fue baja (alfa de Cronbach 0,23). 
Esto se debe, según Goode y Hatt, a lo siguiente: a) diferencias de conocimiento; b) heterogeneidad de los sujetos; y, c) redacción de las preguntas (22). Respecto a las dos primeras razones, la explicación sería que la formación ética de los internos varía dependiendo de la escuela de medicina de origen.

\section{AGRADECIMIENTOS}

A los doctores León Llacsa Soto (Ica), Roger Haro P (Trujillo), Fernando Correa Tineo y Fernando Mendoza Canales (Huancayo) por su colaboración; y a los internos que participaron en el estudio y permitieron llevar a cabo este estudio.

Estudio con Asignación del VII Concurso de proyectos de investigación de la Facultad de Medicina, UNMSM.

\section{REFERENCIAS BIBLIOGRÁFICAS}

1. Fernández C. La responsabilidad civil del médico y el consentimiento informado. Lima: Motivensa Editora Jurídica, 2011.

2. Elwyn G, Edwards A, Kinnersley P. Shared decisionmaking in primary care: the neglected second half of the consultation. Brit J Gen Pract. 1999;49:477-82.
3. Savage R, Armstrong D. Effect of a general practitioner's consulting style on patients' satisfaction: a controlled study. BMJ. 1990;301:968-70.

4. Brock DW, Wartman SA. Sounding board. When competent patient make irrational choices. N Engl J Med. 1991;322(22):1595-9.

5. Pellegrino ED. Relationship between autonomy and integrity in medical ethics. Bol Of Sanit Panam. 1990;108(5-6):379-90.

6. Quill TE, Brody H. Physician recommendation and patient autonomy: finding a balance between physician power and patient choice. Ann Intern Med. 1996;125:763-9.

7. O'Connor AM, Rostom A, Fiset V, Tetroe J, Entwistle $\mathrm{V}$, Llewellyn-Thomas $\mathrm{H}$, Holmes-Rovner M, Barry $M$, Jones J. Decision aids for patients facing health treatment or screening decisions: systematic review. BMJ. 1999;319:731-4.

8. Jonsen AR, Siegler M, Winsdale WJ. Clinical Ethics: A Practical Approach to Ethical Decisions in Clinical Medicine. Sixth Edition. New York: McGraw-Hill, 2006.

9. Kleiman I. Editorial. The right to refuse treatment: ethical considerations for the competent patient. Can Med Assoc J. 1991;144(19):1219-22.

10. Zinn WM. Doctors Have Feeling Too. JAMA. 1988;259:3296-8.

11. Huijer M, van Leeuwen E. Personal values and cancer treatment refusal. J Med Ethics. 2000;26:35862.

12. Consejo Nacional de Salud. Comité de bioética. Guía para la confección de un documento de consentimiento informado en la práctica clinica. Mimeografiado. Lima, 2009.

13. Connelly JE. Refusal of treatment. En: Sugarman J. Ethics in Primary Care. New York: Mc-Graw Hill, 2000:187-98.

14. Friedman Ross L, Lantos J. Treating minors. En: Sugarman J. Ethics in Primary Care. New York: Mc-Graw Hill, 2000:177-85.
15. Bascom P, Tolle SW. Treatment at the end of life. En: Sugarman J. Ethics in Primary Care. New York: Mc-Graw Hill, 2000:211-20.

16. Beauchamp TL, Childress JF. Principles of Biomedical Ethics. Sixth edition. New York: Oxford University Press, 2009.

17. Congreso de la República. Ley General de Salud N 26842. El Peruano 20 de julio de 1997.

18. Gracia D. La tradición médica y el criterio del bien del enfermo. El paternalismo médico. En: Fundamentos de bioética. Madrid: Editorial EUDEMA. 1989:23-107.

19. American Academy of Pediatrics, Committee of Bioethics. Religious Objections to Medical Care. Pediatrics. 1997;99(2):279-81.

20. Emanuel EJ, Emanuel LL. Proxy decision making for incompetent patients. JAMA. 1992;267(15):206771.

21. Zavala S, Sogi C, Cárdenas M, Delgado A. Autonomía del paciente y derecho a rehusar tratamiento: conocimiento en médicos jóvenes peruanos. (En prensa).

22. Goode WJ, Hatt PK. Métodos de investigación social. México: Editorial Trillas, 1988.

Artículo remitido el 4 de noviembre de 2011 y aceptado para publicación el 3 de enero de 2012.

Correspondencia:

Dra. Cecilia Sogi Uematsu

Correo electrónico: ceciliasogi@yahoo.com 